February 9, 2020 10:28 WSPC/INSTRUCTION FILE ws-ijmpe-quarksaturation

International Journal of Modern Physics E

(C) World Scientific Publishing Company

\title{
Looking for quark saturation in proton and nuclei
}

\author{
Wei Zhu \\ Department of Physics, East China Normal University, Shanghai 200062, P.R. China \\ wzhu@phy.ecnu.edu.cn \\ Rong Wang \\ Institute of Modern Physics, Chinese Academy of Sciences, Lanzhou 730000, P.R. China \\ rwang@impcas.ac.cn \\ Jianhong Ruan \\ Department of Physics, East China Normal University, Shanghai 200062, P.R. China \\ jhruan@phy.ecnu.edu.cn \\ Received Day Month Year \\ Revised Day Month Year
}

The quark saturation behavior at low $Q^{2}$ is shown in a numeric solution of the DGLAP equation with parton recombination corrections, which resembles the widely discussed JIMWLK saturation of gluons. Our calculation suggests that the partonic saturation can be interpreted as a dynamical balance between the splitting and the fusion processes of partons, without any other condensation mechanisms added. The nuclear shadowing saturation at small $x$ resulted from the proposed quark saturation is also discussed.

Keywords: quark saturation; nuclear shadowing saturation.

PACS numbers: 12.38. Bx, 12.40.Vv, 13.60.Hb, 25.30.Fj

\section{Introduction}

The Jalilian-Marian-Iancu-McLerran-Weigert-Leonidov-Kovner (JIMWLK) equation ${ }^{1 / 5}$ sums the contributions of multi-gluon fusions to the Balitsky-Fadin-KuraevLipatov (BFKL) evolution. [6 11 An interesting property of JIMWLK equation is a flattish solution of the unintegrated gluon distribution $\phi_{g}\left(x, k_{T}\right)$ in the region of small transverse momentum $k_{T}<Q_{s}$, in which $Q_{s}$ is commonly referred as the saturation scale. Assuming that the transition into the saturation region occurs abruptly near $k_{T} \sim Q_{s}$, one can find that the majority of gluons in this saturation solution have transverse momentum $k_{T} \sim Q_{s}$. This special solution is speculated as a new state of matter - the color glass condensation (CGC), which has been discussed massively. However, the nature of the CGC is still unclear.

Generally, the partonic saturation implies that the occupation of partons in a fast proton reaches a limit and the number of partons stops growing up. To es- 
timate the saturation scale simply, one can apply the following naive picture ${ }^{12}$ The integrated parton distributions $x f\left(x, Q^{2}\right)$ at scale of $Q^{2}$ is the number of partons per unit rapidity interval (the parton rapidity is defined as $y=\ln (1 / x)$ and $\left.x f\left(x, Q^{2}\right)=d N / d y\right)$. Therefore the transverse space per parton is $\pi R_{N}^{2} / x f\left(x, Q^{2}\right)$ in a nucleon of radius $R_{N}$, and the cross section for two parton interaction is estimated to be $C \alpha_{s} / Q^{2}$, in which $C$ is a model-dependent factor. The saturation effect is important when the number of partons per unit of rapidity times the gluon-gluon interaction cross section approaches the geometric size of the nucleon. We call it a full occupation limit. Under this assumption, the corresponding scale $Q_{s}$ can be estimated by

$$
Q_{s}^{2}=C \alpha_{s} \frac{x f\left(x, Q_{s}^{2}\right)}{\pi R_{N}^{2}} .
$$

The saturation critical boundary $Q_{s}(x)$ separates the dilute and dense partonic systems in the $\left(x, Q^{2}\right)$ plane. Assuming $x f\left(x, Q^{2}\right)=\pi R_{N}^{2} Q^{2} /\left(C \alpha_{s}\right)$ is required at $Q<Q_{s}$, the resulting flat distribution of $x f\left(x, Q^{2}\right)$ is a clear feature of the saturation. Note that it is difficult to determine the value of $Q_{s}$ in the experiment because the transition into saturation may not occur abruptly, even though $Q_{s}$ can be given in a detailed calculation. From above estimation, the following saturation features are given, which are useful in searching/discriminating the partonic saturation in experiments. (i) The distribution is strong $Q^{2}$-dependent at $Q<Q_{s}$, which is written as $x f\left(x, Q^{2}\right) \sim \alpha_{s}^{-1} Q^{2}$, while it is weakly $Q^{2}$-dependent at $Q>Q_{s}$ according to the standard linear QCD evolution equations, which is written as $x f\left(x, Q^{2}\right) \sim \ln \left(Q / \Lambda_{Q C D}\right)$; (ii) The values of $x f\left(x, Q^{2}\right)$ at $Q<Q_{s}$ are $x$-independent if $x$ enters the sea quark dominated region; (iii) The corresponding unintegrated parton distribution $k_{T} \phi\left(x, k_{T}^{2}\right)$ has a peak at $k_{T} \sim Q_{s}$ (the integrated parton distribution $x f\left(x, Q^{2}\right)$ is the $k_{T}$ integrated distribution of $\phi\left(x, k_{T}^{2}\right)$, and here we have $\left.\phi\left(x, k_{T}^{2}\right) \simeq \partial x f\left(x, Q^{2}\right) /\left.\partial Q^{2}\right|_{Q^{2}=k_{T}^{2}}\right)$.

The deep inelastic scattering (DIS) data at small $x$ and low $Q^{2}$ is an interesting domain for high energy physics, where the partons have a larger correlation length $\sim 1 / Q$ and stronger recombination strength. Especially, more attention should be paid to small $x$ and low $Q^{2}$ for searching the parton saturation. In this paper, we try to look for the possible quark saturation at low $Q^{2}$. In our previous work, the parton distributions in the proton and nuclei are dynamically generated from a extreme low resolution scale where the nucleon have merely valence quarks by using a nonlinear QCD evolution equation - the DGLAP equation with the recombination corrections 15 We find that the produced sea quark distributions present a positive and flat distribution in the region of small $x$ and low $Q^{2}$. It is surprising to find that the saturation behaviors are also shown for the quark distributions, which will be detailed in Sec. 2 ,

At low $Q^{2}$ scale, the naive parton distributions partly contribute to the measured structure functions. The nonperturbative QCD contributions are not negligible to the structure functions, which make it difficult to look for the possible quark sat- 


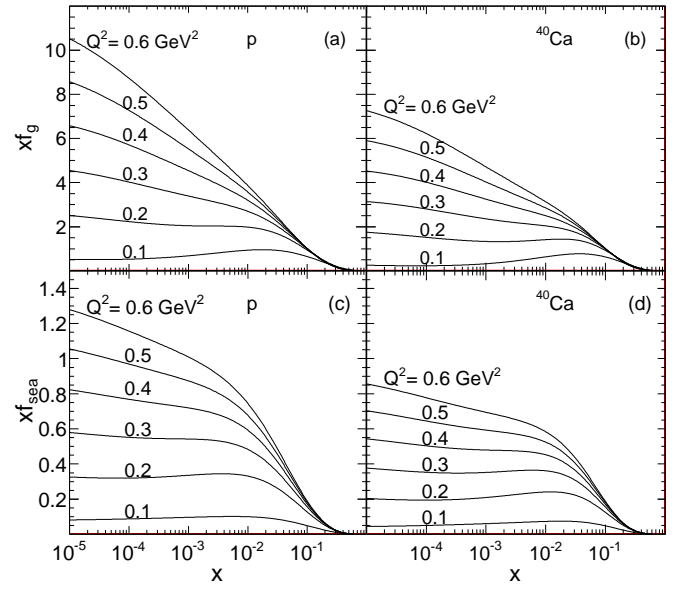

Fig. 1. Our predicted parton distributions in the low $Q^{2}$ range, where the platform-like distributions are found for sea quarks at very low scale.

uration signature at low $Q^{2}$. In order to identify the quark saturation from the experimental data, we need to subtract the nonperturbative contributions from the measured structure function $F_{2}^{p}\left(x, Q^{2}\right)$ at $Q^{2}<1 \mathrm{GeV}^{2}$. The popular phenomenological model - the vector meson dominance (VMD) mode $1 \frac{18}{20}$ is used to mimic these nonperturbative corrections, which is described in Sec. 3 .

In order to confirm the suggested quark saturation for free proton above, we also investigate the previous experimental data of nuclear shadowing effect at small $x$ and low $Q^{2}$. The nuclear shadowing saturation resulted from the quark saturation in nuclei, a flat distribution of structure function ratio at small $x$ and low $Q^{2}$, is discussed in Sec. 4. Moreover, it is found that the nuclear shadowing effect has different $Q^{2}$-dependent behaviors inside and outside of the saturation domain. More precise experiments at low $Q^{2}$ are needed to verify the quark saturation. Discussions and summary are given in Sec. [5.

\section{A solution of quark saturation in nonlinear QCD evolution equation}

The physics of parton distributions at low $Q^{2}$ are rarely discussed. Nevertheless it is an important and interesting topic as it is related to the origins of sea quarks and gluons at high $Q^{2}$. In our previous work, the gluon and sea quark distributions in the proton at $Q^{2}<1 \mathrm{GeV}$ are given by the nonlinear evolution equation, $15-17$ which are shown in Fig. 1. Fig. 1(c) presents one interesting saturation feature of sea quarks. The sea quark distribution $x f_{\text {sea }}\left(x, Q^{2}\right)$ reach a plateau at $x<0.01$ and $0.1 \mathrm{GeV}^{2}<Q^{2}<0.6 \mathrm{GeV}^{2}$, and the height of the plateau linearly goes up with $Q^{2}$ increasing. 

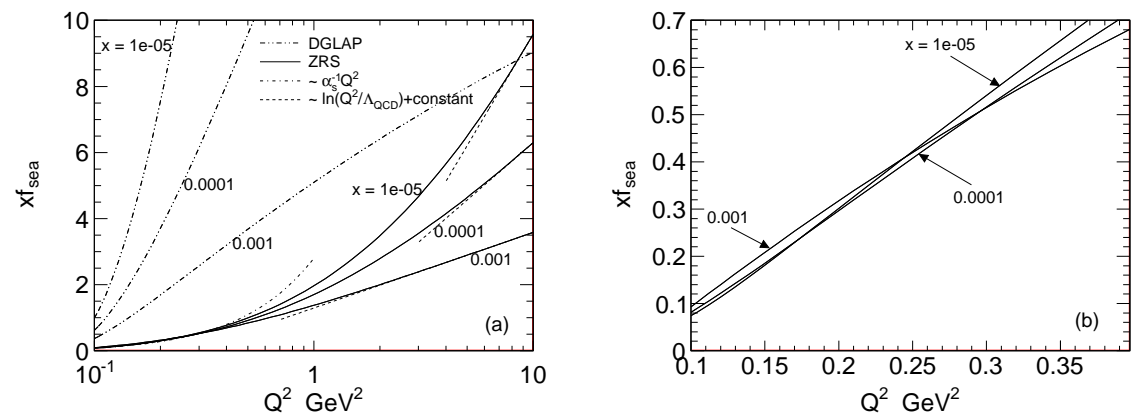

Fig. 2. Our predicted proton structure functions (solid curves) at low $Q^{2}$ and small $x$. The asymptotic curves of the $Q^{2}$-dependence of the structure function $F_{2}$ are shown: function form $x f_{\text {sea }} \sim \alpha_{s}^{-1} Q^{2}$ (dash-dot curve) at low $Q^{2}$ and function form $x f_{\text {sea }} \sim \ln Q^{2}$ (dash curve) at high $Q^{2}$. The dash-dot-dot curves are the solutions of the linear DGLAP equation. The transition between the two asymptotic behaviors is one main characteristic of the parton saturation.

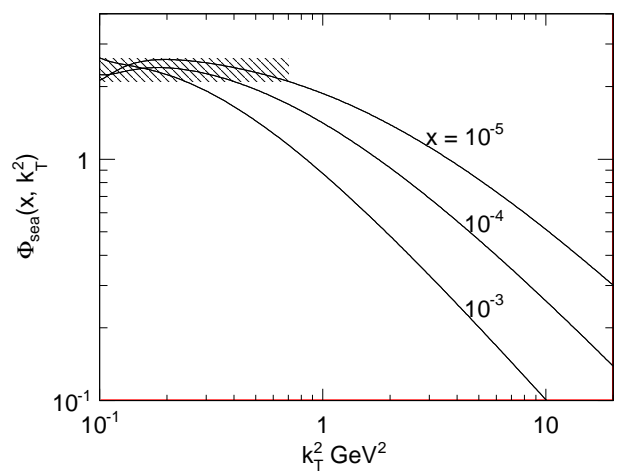

Fig. 3. Unintegrated sea quark distributions in the proton at low $Q^{2}$. The cloud square presents the quark saturation domain.

Two asymptotic function $x f_{\text {sea }}\left(x, Q^{2}\right) \propto \alpha_{s}^{-1} Q^{2}$ and $x f_{\text {sea }}\left(x, Q^{2}\right) \propto \ln Q^{2}$ are shown together with our predicted sea quark distribution as a function of $Q^{2}$ in Fig. 2, to demonstrate the different $Q^{2}$-dependence at low and high $Q^{2}$. Sea quark distributions $x f_{\text {sea }}\left(x, Q^{2}\right)$ generated by the DGLAP $21 \sqrt{23}$ evolution are also shown. The obvious difference between the result by nonlinear evolution and that by the DGLAP evolution indicates that the nonlinear effect is substantial in low $Q^{2}$ range. It is easy to find the saturation features (i) and (ii) discussed in Sec. 1 from our predicted quark distributions. The coincidence of the predicted parton distributions at different $x$ shown in Fig. 2(b) implies a geometrical scaling behavior $x f_{\text {sea }}\left(x, Q^{2}\right)=\psi\left(Q^{2} / \Lambda_{Q C D}^{2}\right)$.

A more clear demonstration of the quark saturation solution presented in Fig. 


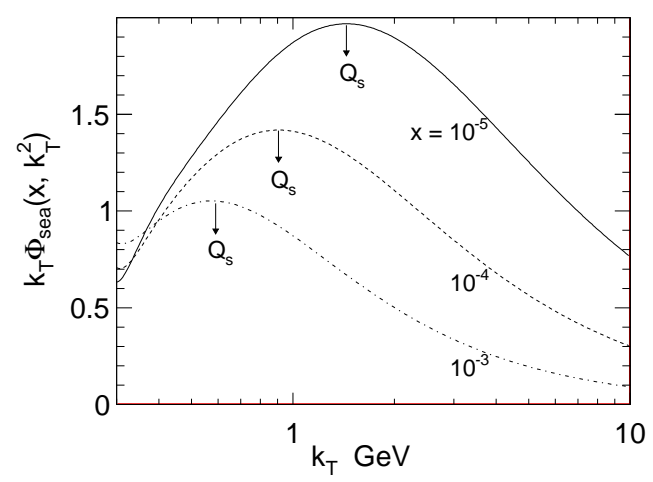

Fig. 4. Unintegrated sea quark distributions in the proton (multiplied by the phase space factor of the quark transverse momentum $k_{T}$ ) as a function of $k_{T}$. The quark momentum accumulation near $k_{T} \sim Q_{s}$ is a characteristic of quark saturation.

3. is the unintegrated quark distribution $\left.\phi_{\text {sea }}\left(x, k_{T}^{2}\right) \simeq\left[\partial x f_{\text {sea }}\left(x, Q^{2}\right) / \partial Q^{2}\right]\right|_{Q^{2}=k_{T}^{2}}$, where the contributions of the Sudakov factor are neglected at very small $x . \underline{[24}$ Note that our resulting curves are determined by the data at high $Q^{2}$, which have some errors derived from the experimental errors. A small uncertainty in $f_{\text {sea }}\left(x, Q^{2}\right)$ calculation results in some obvious undulation in $\phi_{\text {sea }}\left(x, k_{T}^{2}\right)$. Hence in the geometrical scaling range, the parton distributions at different $x$ are not ideally equal (Fig. 2(b)). In Fig. 3, the unintegrated quark distributions in the saturation domain vary in a narrow range (the shadowed block shown in Fig. 3), instead of a plateau line predicted by the JIMWLK equation.

Our predicted unintegrated quark distribution $k_{T} \phi_{\text {sea }}\left(x, k_{T}^{2}\right)$ (multiplied by the phase space factor of the quark transverse momentum $k_{T}$ ) as a function of $k_{T}$ is shown in Fig. 4, showing that the major sea quarks have momentum of $k_{T} \sim Q_{s}$. We suggest to call the peak which is of the largest occupation numbers the quark saturation rather than the condensation. It is also found that the transition from the normal diluted parton state to the saturation limit occurs though a rather broad range of $Q^{2}$, i.e., the transition into the saturation does not occur abruptly. For reference, similar distributions for the gluon density in the GBW (Golec-Biernat Wüsthoff) mode ${ }^{25 \mid 26}$ are shown in Fig. 5. This model is generally used to simulate the JIMWLK gluon saturation. Note that the gluon distribution by GBW lacks $1 / k_{T}$ tail. The reason is that the parton evolution is not treated in this model.

A surprise is that there is no obvious saturation features for the gluon distribution in the low $Q^{2}$ range (See Fig.1(a)), although the recombination effect for gluon distribution is much stronger than that for sea quark distributions as the two leading nonlinear corrections are both about gluons $P_{g g \rightarrow g g} \sim 1 / x$ and $P_{q \bar{q} \rightarrow g g} \sim 1 / x$ at small $x ! \frac{15] 17}{17}$ The absence of gluon saturation at low $Q^{2}$ can be understood as follows. The main source of gluons near the starting evolution scale is the radiation 


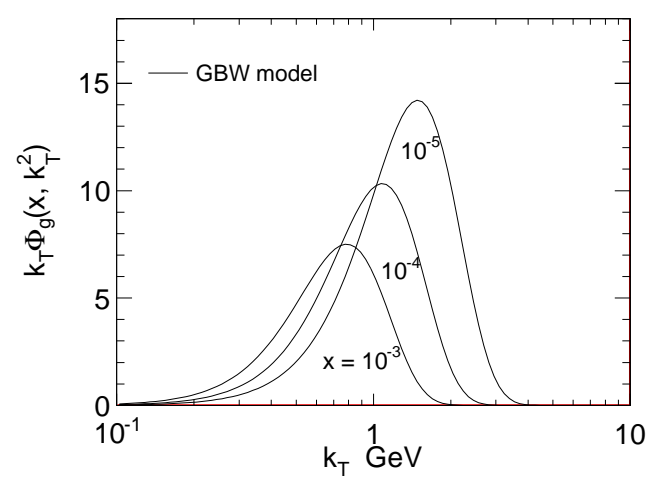

Fig. 5. A typical unintegrated gluon distribution simulated by the GBW model $\sqrt[25]{26}$ Note that the GBW gluon lacks a $1 / k_{T}$ tail since the parton evolution is not applied in this model.

of valence quarks. The newly generated gluons from valence quarks break the possible saturation balance between gluon splitting and gluon combining. This situation is different from the JIMWLK saturation, where the gluon radiation from valence quarks is overwhelmed by the gluon splitting at small $x$. Away from the starting scale, the number of gluons grow very fast and dominates at small $x$. The radiated gluons directly from the valence quarks is relatively of small quantity at high $Q^{2}$ scale.

The phenomenon of the gluon saturation widely discussed is known as the CGC. Current theoretical understanding suggests that the CGC is the collective gluon excitations based on the thought that the JIMWIK equation sums all orders of gluon fusions. However, the quark saturation from nonlinear evolution equation has the similar features of the CGC, which implies that the partonic (gluon and quark) saturation is a dynamical balance between strong splitting and fusion processes rather than a new condensation mater state. The origin of partonic (gluon and quark) saturation does not need any condensation dynamics.

\section{Quark saturation shown in the proton structure function at low $Q^{2}$}

Is the quark saturation only a mathematic solution in a nonlinear QCD evolution equation, or a real physical existence? To answer this question, we should look for the observable patterns of the quark saturation in experiments. Parton distribution functions are extracted from the measured structure functions, however the nonperturbative QCD components are mixed with the possible saturation signature at low $Q^{2}$. In fact, at low $Q^{2}$, the multi-parton correlations dominate, and the inclusive lepton-nucleon cross section is mainly from the complicate higher twist interactions. According to the operator product expansion (OPE), the proton structure function 


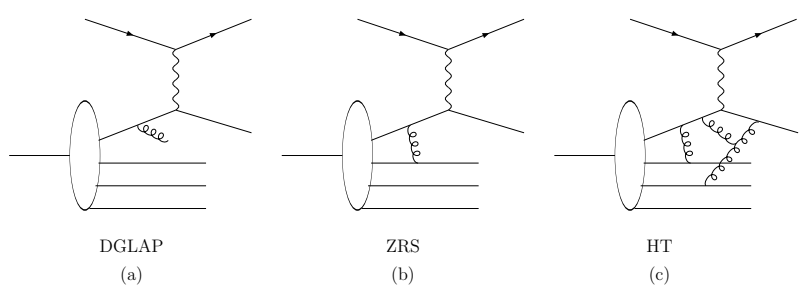

Fig. 6. DIS processes. (a) The leading twist contributions; (b) A twist-4 corrections; (c) The high twist contributions, which can be isolated by using a naive VMD model.

$F_{2}^{p}\left(x, Q^{2}\right)$ can be written as a series in $1 / Q^{2}$,

$$
F_{2}^{p}\left(x, Q^{2}\right)=F_{2}^{L T}\left(x, Q^{2}\right)+F_{2}^{H T}\left(x, Q^{2}\right) .
$$

The leading (twist-2) term corresponds to scattering from one single free parton, while higher twist terms correspond to multi-parton interactions. Up to date, only a part of higher twist effects, for example, the recombination of initial partons (Fig. 6(b)) has been calculated perturbatively as the corrections to DGLAP evolution, which have been discussed in the previous section. We denote this single parton scattering contribution to the structure function as $F_{2}^{D G L A P+Z R S}\left(x, Q^{2}\right)$. However, we can neither perform nor interpret a partonic calculation of higher twist effects of the correlations between the initial and finite partons, since they break the factorization schema. In a certain kinematic region, some of such higher twist contributions to $F_{2}^{p}\left(x, Q^{2}\right)$ appear to be some hadronic interaction phenomena. For this situation, we may chose a suitable phenomenological model to describe the corresponding higher twist effects.

We try to use the well known Vector Meson Dominance (VMD) model to mimic the mentioned higher twist corrections above. The contributions shown in Fig. 6(c) can not be neglected at low $Q^{2}$. The corrections of the quark-antiquark pair is needed, which interacts with the target like a virtual vector meson if the transverse momentum $k_{\perp} \sim Q$ of the quark pair is not large, and the confinement effects play significant roles. Since contribution shown in Fig. 6(c) can not be factorized, we apply a phenomenological VMD model (Fig. 7) to calculate it. Traditionally, the VMD mode ${ }^{27 \mid 28}$ was used to explain the structure function in low $Q^{2}$ region successfully. We denote this contribution as $F_{2}^{V M D}\left(x, Q^{2}\right)$.

In the VMD model,

$$
F_{2}^{V M D}\left(x, Q^{2}\right)=\frac{Q^{2}}{4 \pi \gamma_{\rho}^{2}} \frac{m_{\rho}^{4} \sigma_{\rho p}}{\left(Q^{2}+m_{\rho}^{2}\right)^{2}} .
$$

where $\gamma_{\rho}$ is the coupling constant between $\rho$ vector meson and proton, $x$ is a variable defined as $x=Q^{2} /\left(s+Q^{2}-m_{p}^{2}\right)$ rather than the momentum fraction of a parton, and $s$ is the center-of-mass energy (W) square of the $\gamma p$ collision. We considered the contributions of $\rho$ meson only, because it is the dominant virtual particle involved. 


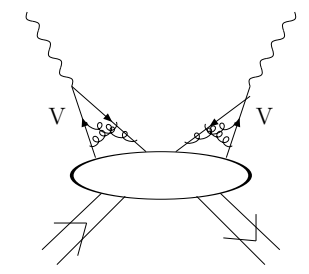

Fig. 7. The diagram of VMD model.

The cross sections $\sigma_{\rho p}(W)$ is the total cross section of the virtual $\rho$ meson scattering on the nucleon.

At high energy, the Regge theory ${ }^{29}$ is successful to parameterize the cross sections $\sigma_{\rho p}$. The high energy behavior in Regge theory for the total cross section is expressed as $\sigma_{\rho p}(s) \sim s^{\alpha_{P}-1}$, where $\alpha_{P}=1.0808$ is the intercepts of the Pomeron ${ }^{30}$ At large $1 / x$, there is $\sigma_{\rho p}(x) \sim x^{1-\alpha_{P}}$. The elastic scattering dominates in large $x$ region for $F_{2}^{p}\left(x, Q^{2}\right)$. The contributions of Pomeron is fast reduced with $x$ increasing. We neglect the Regge contribution at large $x$ in this work. In consequence,

$$
F_{2}^{V M D}\left(x, Q^{2}\right) \simeq B \frac{m_{\rho}^{2} Q^{2}}{\left(Q^{2}+m_{\rho}^{2}\right)^{2}} x^{1-\alpha_{P}}(1-x)^{20},
$$

where $B$ is a free parameter and determined to be $B=0.4$ from a fit to the experimental data at low $Q^{2}$. An arbitrary large power in the factor $(1-x)^{p}(p>>$ 1 ) is applied to suppress the contributions of Pomeron at large $x$. Note that the contribution of $F_{2}^{D G L A P+Z R S}\left(x, Q^{2}\right)$ is taken from Ref., $\frac{13}{13}$ which is already fixed and determined by the experimental measurements at high $Q^{2}>4 \mathrm{GeV}^{2}$.

More complicated corrections to $F_{2}^{p}\left(x, Q^{2}\right)$ at low $Q^{2}$ are from the higher order QCD effects $\mathcal{O}\left(\alpha_{s}\right)$ and the higher order recombinations. In principle, we need to consider all these corrections, however it is still worthwhile to look at the leading corrections at the beginning. Our interest is that if higher order corrections are small down to some low scale $Q^{2}\left(\sim \mu^{2}\right)$, then our leading order analysis of structure function data to $\mu^{2}$ is acceptable. Otherwise, our calculation is still important in order to extract the higher order contributions.

Finally, the proton structure function without higher order QCD effects and higher order recombinations is written as

$$
F_{2}^{p}\left(x, Q^{2}\right) \simeq P F_{2}^{D G L A P+Z R S}\left(x, Q^{2}\right)+F_{2}^{V M D}\left(x, Q^{2}\right),
$$

where $P$ is the probability of inelastic events via bare photon-parton interaction. As $m_{\rho}^{4} /\left(Q^{2}+m_{\rho}^{2}\right)^{2}$ in Eq. (44) is the probability of the VMD event, the factor $P$ in Eq. (5) for the single parton scattering is then given by

$$
P=1-\frac{m_{\rho}^{4}}{\left(Q^{2}+m_{\rho}^{2}\right)^{2}} .
$$




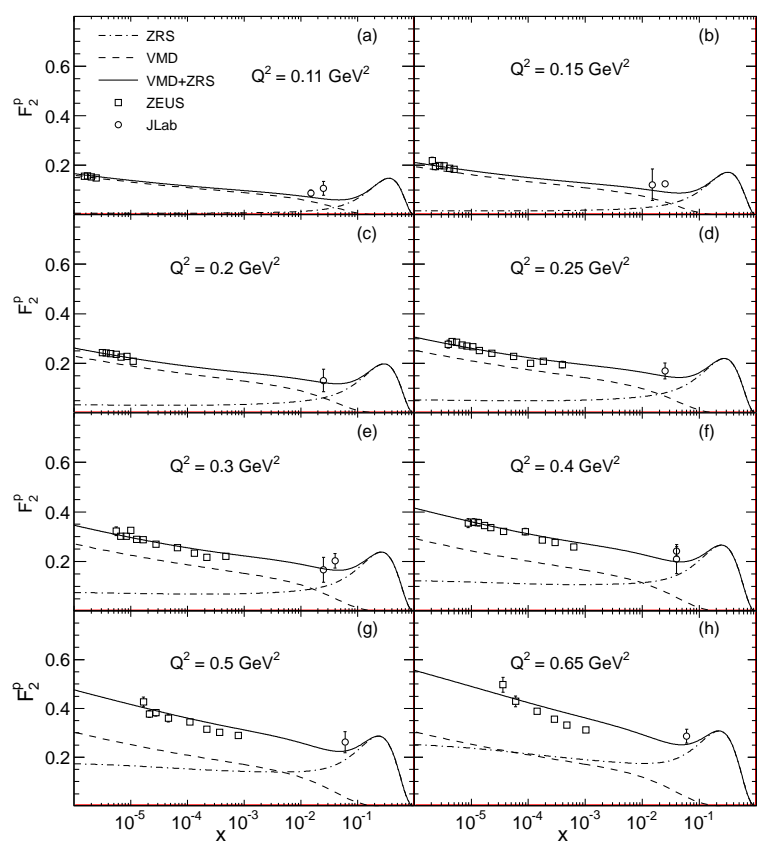

Fig. 8. The proton structure function $F_{2}^{p}\left(x, Q^{2}\right)$ as a function of $x$ at various $Q^{2}\left(<1 \mathrm{GeV}^{2}\right)$. The contributions of $F_{2}^{\text {hadron }}\left(x, Q^{2}\right)$ and $F_{2}^{D G L A P+Z R S}\left(x, Q^{2}\right)$ are shown separately. The data are taken from $[31+36$ One can find that a flattish quark distribution is important to the measured structure function.

The results of our model is compared to experimental measurements $31 \sqrt{36}$ in Fig. 8 (at $Q^{2}<1 G e V^{2}$ ) and in Fig. 9 (at $Q^{2}>1 G e V^{2}$ ). The qualities of the fits are good. Our model about the quark saturation gives consistent results with the observable data.

\section{Quark saturation shown in nuclear shadowing at low $Q^{2}$}

The cross section ratios of inelastic muon scattering on nuclei to that on deuterium at very small $x(0.00002<x<0.25)$ and low $Q^{2}$ down to $0.1 G e V^{2}$ was measured by Fermilab E665 experiment. ${ }^{37] 38}$ The cross section ratio shows a flat distribution at $x<10^{-3}$. This nuclear shadowing saturation was confirmed by the later NMC data $39 \sqrt[30]{40}$ These flat distributions are a sign of the quark saturation.

The saturation observed in the nuclear structure function ratio is related to two different saturation mechanisms. The saturation of $F_{2}^{D G L A P+Z R S}\left(x, Q^{2}\right)$ resulted from the quark saturation has been discussed in Sec. 2. The quark saturation feature is clearly presented for the nuclear quark distributions at small $x, \frac{14}{14}$ which is shown in Fig. 1(d). The other mechanism for the saturation of nuclear structure function ratio is due to the hadron part of the structure function $F_{2}^{V M D}$. The underlying process is the multiple-scattering of virtual meson off nuclei. In the VMD model 


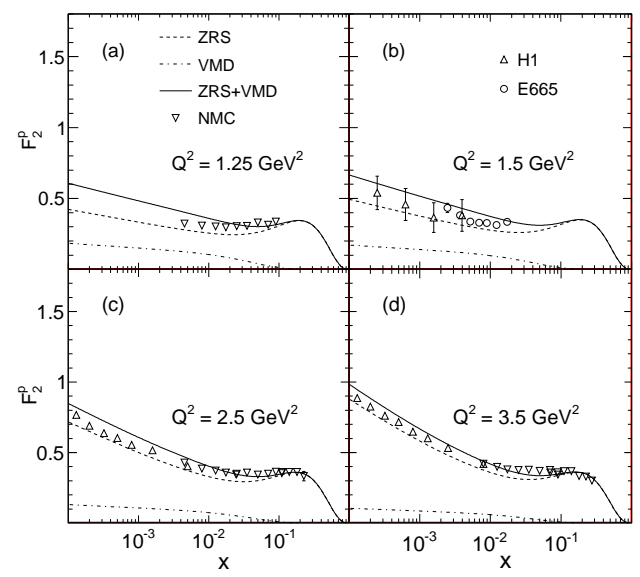

Fig. 9. Same as Fig. 8 but for $Q^{2}>1 G e V^{2}$.

for the nuclei,

$$
F_{2}^{A, V M D}\left(x, Q^{2}\right)=\frac{Q^{2}}{4 \pi \gamma_{\rho}} \frac{m_{\rho}^{4} \sigma_{\rho A}}{\left(Q^{2}+m_{\rho}^{2}\right)^{2}} .
$$

Using the Glauber eikonal approximation, $\frac{41] 42}{42}$ the relation between the total cross sections $\sigma_{V A}$ and $\sigma_{V N}$ is given by

$$
\sigma_{\rho A}=2 \int d^{2} b\left[1-e^{-\sigma_{\rho p} T_{A}(b) / 2}\right] \simeq G\left(R_{A} / l_{V}\right) \sigma_{V p},
$$

where $T_{A}(b)$ is the nuclear thickness function and

$$
G(x)=\frac{3}{x^{3}}\left[(1+x) e^{-x}-1+\frac{1}{2} x^{2}\right],
$$

and $l_{V}$ is the mean free path of the hadronic fluctuation constituent of the virtual photon ${ }^{20}$ It is expected that $l_{V}$ is much larger than the mean free path of the real vector meson in the nucleus. Figure 10 shows the results of nuclear structure function ratios at low $Q^{2}$ using $l_{V}=8 \mathrm{fm}$. There is strong kinematic correlation between $x$ and $Q^{2}$ in the fixed target experiments, which makes the low $x$ measurements also at low $Q^{2}$. Our predicted ratios at $x<10^{-3}$ exhibit a weak dependence on $x$ and $Q^{2}$, which are consistent with the experimental data. However more precise measurements are highly needed. A wide energy and four-momentum transfer range of the future Electron-Ion Collider (EIC) would provide valuable opportunities to search for the quark saturation.

The other possible solution for the structure function ratio saturation is also considered: the shapes of quark distribution functions in proton and nucleus are similar towards small $x$ at large $Q^{2}$ yet not flat. Our predicted $F_{2}^{p}\left(x, Q^{2}\right)$ and $F_{2}^{C a}\left(x, Q^{2}\right)$ at $Q^{2}>>1 G e V^{2}$ are shown in Fig. 11, where the nonlinear corrections 


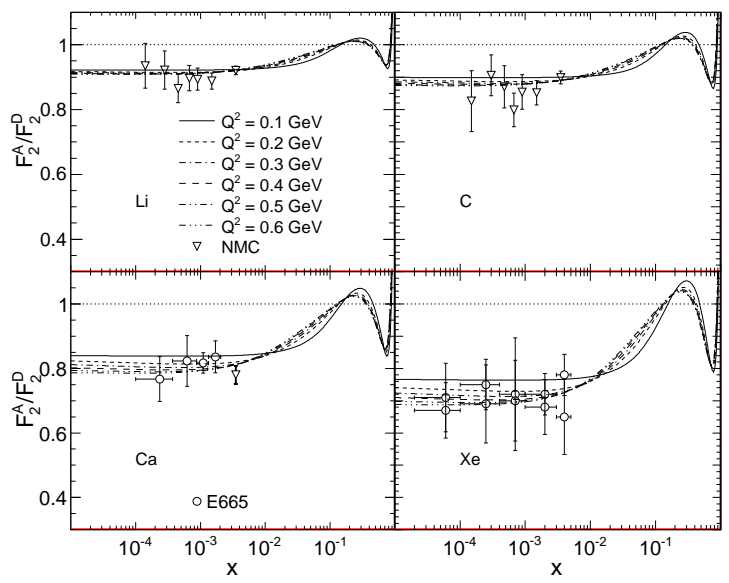

Fig. 10. Predicted ratios of $F_{2}^{A} / F_{2}^{p}$ for several nuclei at $Q^{2}<1 \mathrm{GeV}^{2}$ compared to experimental data 37.40 The quark saturation is important for the observed platform-like distribution.

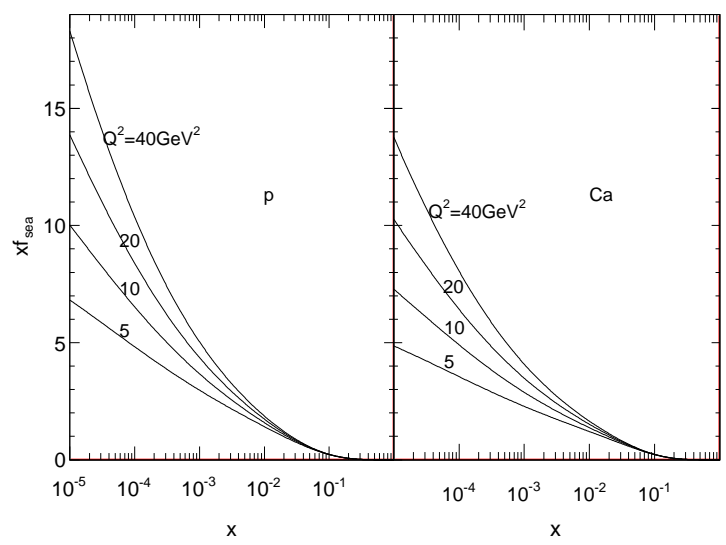

Fig. 11. Sea quark distributions in proton and calcium at $Q^{2}=5-40 \mathrm{GeV}^{2}$.

are negligible. The platform-like distribution is not found at high $Q^{2}$ (see Fig. 12). The $Q^{2}$ - and $x$-dependent behaviors of the nuclear shadowing at high $Q^{2}$ are different from the nuclear shadowing saturation.

\section{Discussions and Summary}

The typical parton evolution configuration of the proton in $\left(x, Q^{2}\right)$ space is shown in Fig. 13. The starting point of the evolution is the proton at extreme low $\mu^{2}$, which is represented as mere three valence quarks. With the increase of the virtuality 


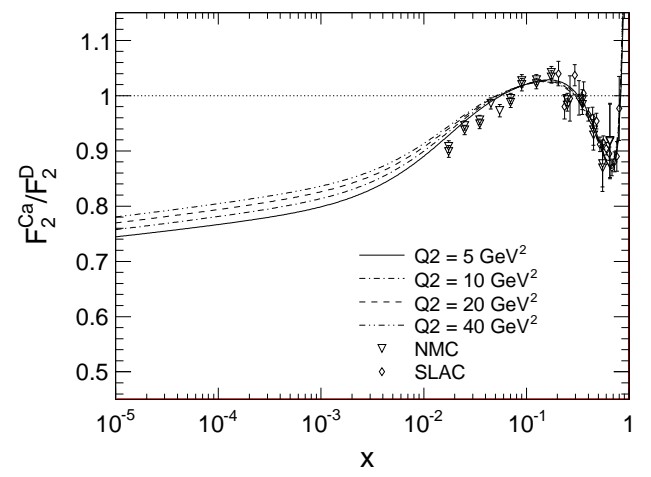

Fig. 12. Predicted ratios for $F_{2}^{C a} / F_{2}^{p}$ at $Q^{2}>>1 \mathrm{GeV}^{2}$. It has different $Q^{2}$ - and $x$-dependence compared to shadowing saturation at low $Q^{2}$.

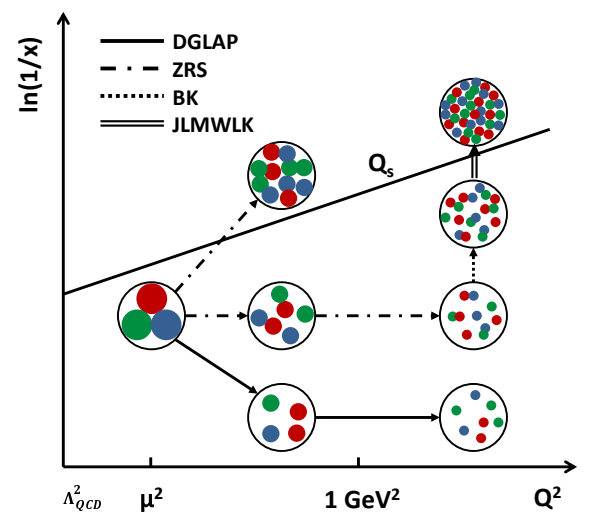

Fig. 13. A schematic diagram for illustrating the different evolution processes of parton distributions in different kinematic regions.

of the probe while the value of $x$ is fixed, we are able to resolve the partons into smaller ones. This type of evolution is well described by the DGLAP equation at relatively large $x$, and the DGLAP equation including parton recombination corrections at small $x$. In the first case, the number of partons rises logarithmically while their typical size decreases like $\alpha_{s} / Q^{2}$ so that the partons in the proton become more and more dilute. However in the second case, the number of sea quarks rises as $\sim Q^{2} / \alpha_{s}$, and it keeps in the full occupation situation (splitting and fusion balance), which leads to the quark saturation. In a little larger $Q^{2}$ but very small $x$ range, BFKL evolution with gluon recombination, namely the BK evolution (with leading recombination) and JIMWLK evolution (with multi-recombinations) towards smaller $x$ with $Q^{2}$ fixed are commonly used.

Different evolution schemes have different kinds of saturations. The proton in the 
JIMWLK evolution process leads to the black-disc limit, for which the gluons are fully overlapped, namely, the JIMWLK saturation. The three-gluon recombination process of JIMWLK equation plays an important role in the gluon saturation. The quark saturation in low $Q^{2}$ originates from the leading order parton recombinations with big strong coupling constant. The saturation for the quark distributions can not be directly probed in the deep inelastic scattering at low $Q^{2}$, because the hadronic component of the probe is mixed with the quark saturation. The gluon saturation by the JIMWLK equation is the result of multi-recombination of gluons at high $Q^{2}$, which is also can not be observed directly by the electro-magnetic probe. It is very interesting that these two kinds of saturation have similar features, which suggests that both the quark saturation and the gluon saturation comes from the dynamic balance between the parton recombination and parton splitting.

In summary, a numeric solution of the DGLAP equation with parton recombination corrections at low $Q^{2}$ shows the quark saturation, which likes the JIMWLK saturation of gluons. This calculation shows that the partonic saturation is the result of the dynamical balance between parton splitting and fusion in the context of perturbative QCD theory. The observed nuclear shadowing saturation interpreted as the quark saturation is discussed. We argue that the nuclear shadowing saturation measured by E665 and NMC collaborations is the sign of the quark saturation.

\section{References}

1. J. Jalilian-Marian, A. Kovner, L. McLerran, and H. Weigert, Phys. Rev. D 55 (1997) 5414 .

2. J. Jalilian-Marian, A. Kovner, A. Leonidov, and H. Weigert, Nucl. Phys. B 504 (1997) 415.

3. H. Weigert, Nucl. Phys. A 703 (2002) 823.

4. E. Iancu, A. Leonidiv, and L. McLerran, Nucl. Phys. A 692 (2001) 583.

5. E. Iancu, A. Leonidiv, and L. McLerran, Phys. Lett. B 510 (2001) 133.

6. L.N. Lipatov, Sov. J. Nucl. Phys. 23 (1976) 338.

7. V.S. Fadin, E.A. Kuraev, L.N. Lipatov, Phys. Lett. B 60 (1975) 50.

8. E.A. Kuraev, L.N. Lipatov, V.S. Fadin, Sov. Phys. JETP 44 (1976) 443.

9. E.A. Kuraev, L.N. Lipatov, V.S. Fadin, Sov. Phys. JETP 45 (1977) 199.

10. I.I. Balitsky, L.N. Lipatov, Sov. J. Nucl. Phys. 28 (1978) 822.

11. I.I. Balitsky, L.N. Lipatov, JETP Lett. 30 (1979) 355.

12. L.V. Gribov, E.M. Levin and M.G. Ryskin, Phys. Rep. 100 (1983) 1.

13. X.R. Chen, J.H. Ruan, R. Wang, P.M. Zhang and W. Zhu, Int. J. Mod. Phys. E 23 (2014) 1450057, arXiv:1306.1872 [hep-ph].

14. X.R. Chen, J.H. Ruan, R. Wang, P.M. Zhang and W. Zhu, Int. J. Mod. Phys. E 23 (2014) 1450058, arXiv:1306.1874 [hep-ph].

15. W. Zhu, Nucl. Phys. B 551 (1999) 245, arXiv:hep-ph/9809391

16. W. Zhu, J.H. Ruan, Nucl. Phys. B 559 (1999) 378, arXiv:hep-ph/9907330v2.

17. W. Zhu and Z.Q. Shen, HEP \& NP 29 (2005) 109, arXiv:hep-ph/0406213 3.

18. J.J. Sakurai, in currents and mesons, (The University of Chicago Press, Chicago, 1969).

19. T. H. Bauer, R. D. Spital, D. R. Yennie, and F. M. Pipkin, Rev. Mod. Phys. 50 (1978) 261. 
February 9, 2020 10:28 WSPC/INSTRUCTION FILE ws-ijmpe-quarksaturation

20. G. Grammer and J. D. Sullivan, in Electromagnetic Interactions of Hadrons (Vol. 2), ed. by A. Donnachie and G. Shaw, (Plenum Press, New York, 1978).

21. G. Altarelli and G. Parisi, Nucl. Phys. B 126 (1977) 298.

22. V.N. Gribov and L.N. Lipatov, Sov. J. Nucl. Phys. 15 (1972) 438.

23. Yu.L. Dokshitzer, Sov. Phys. JETP. 46 (1977) 641.

24. M.A. Kimber, A.D. Martin and M.G. Ryskin, Phys. Rev. D 63 (2001) 114027.

25. K. J. Golec-Biernat and M. Wüsthoff, Phys. Rev. D 59 (1998) 014017.

26. K. J. Golec-Biernat and M. Wüsthoff, Phys. Rev. D 60 (1999) 114023.

27. B. Badelek, J, Kwiecinski, B. Ziaja, Eur. Phys. J. C 26 (2002) 45.

28. B. Badelek, J, Kwiecinski, B. Ziaja, Acta Phys. Polon. B 33 (2002) 3701, arXiv:hep-ph/0207325.

29. P.D.B. Collins, in An Introduction to Regge Theory and High Energy Physics, (Cambridge University Press, Cambridge, 1977).

30. A. Donnachie and P. V. Landshoff, Physics Letters B 437 (1998) 408.

31. J. Breitweg, et al. (ZEUS Collaboration), Phys. Lett. B 487 (2000) 53, arXiv:hep-ex/0005018 2 .

32. V. Tvaskis et. al., Phys.Rev. C 81 (2010) 055207.

33. M. Arneodo, et al. (New Muon Collaboration), Nucl. Phys. B 483 (1997) 3.

34. S. Aid, et al. (H1 Collaboration), Nucl. Phys. B 470 (1996) 3, arXiv:hep-ex/9603004

35. C. Adloff et al. (H1 Collaboration), Eur. Phys. J. C 21 (2001) 33.

36. M.R. Adams et al., Phys. Rev. D 54 (1996) 3006.

37. M.R. Adams, et. al., Phys. Rev. Lett. 68 (1992) 3266.

38. M.R. Adams, et. al., Z. Phys. C 67 (1995) 403, arXiv:hep-ex/9505006

39. M. Arneodo et al. (New Muon Collaboration), Nucl. Phys. B 441 (1995) 12.

40. P. Amaudruz et al. (New Muon Collaboration), Zeit. Phys. C 51 (1991) 387.

41. R.J. Glauber, in Lectures in theoretical physics, ed. by W.E. Brittin et al. (Interscience Publishers, New York, 1959).

42. R.J. Glauber and G. Matthiae, Nucl. Phys. B 21 (1970) 135. 